\title{
Different techniques in robotic lung resection
}

\author{
Pierluigi Novellis ${ }^{1}$, Marco Alloisio ${ }^{1,2}$, Umberto Cariboni ${ }^{1}$, Giulia Veronesi ${ }^{1}$ \\ ${ }^{1}$ Division of Thoracic and General Surgery, Humanitas Clinical and Research Center, Rozzano (Milan), Italy; ${ }^{2}$ Biomedical Science Department, \\ Humanitas University, Rozzano (Milan), Italy \\ Correspondence to: Pierluigi Novellis. Division of Thoracic and General Surgery, Humanitas Clinical and Research Center, Via Alessandro Manzoni \\ 56, 20089 Rozzano, Milano, Italy. Email: pierluigi.novellis@cancercenter.humanitas.it. \\ Provenance: This is an invited Editorial commissioned by Section Editor of JTD, Jianfei Shen, MD (Department of Cardiothoracic Surgery, Taizhou \\ Hospital of Zhejiang Province, Wenzhou Medical University, Taizhou, China). \\ Comment on: Jin R, Yang S, Wei G, et al. Robotic Thoracic surgery: left inferior lobectomy. AME Med J 2017;2:13.
}

Submitted Sep 29, 2017. Accepted for publication Oct 10, 2017.

doi: $10.21037 /$ jtd.2017.10.69

View this article at: http://dx.doi.org/10.21037/jtd.2017.10.69

\section{Introduction}

We read with great interest the article of Jin and co-authors describing a left lower lung lobectomy performed with robotic technique (1). The robotic approach for surgical treatment of lung cancer was first introduced in 2002 (2). The initial spread of this technique has been slow because many limitations were emphasized, such as the spatial footprint of the apparatus, the complexity in installing the robot's arms into the patient's chest and the increased duration of surgery; operating at a distance from the patient was also considered a source of anxiety by many surgeons. As a result, time was needed to gain confidence with the new apparatus and change the surgeons' mentality for accepting the new procedure.

In 2009 the database of the Agency for Healthcare Research and Quality (AHRQ, http://hcupnet.ahrq.gov) reported that $66 \%$ of lobectomies were performed via thoracotomy, 33\% via video-assisted thoracoscopic surgery (VATS) and only $1 \%$ with a robotic system; nevertheless in 2013 , the percentages changed the figures were $56 \%, 33 \%$ and $11 \%$, respectively. A recent market analysis conducted in US in 2015 reported that lobectomies performed by robot reached the $15 \%$.

Principal limitations to the wide adoption of robotic thoracic surgery consist in the high capital and running costs of the robot instruments (3). Furthermore it would seem that the use of robotic surgery in general has not improved patient outcomes $(4,5)$, so it is important to provide a balanced assessment between advantages and disadvantages of robot-assisted surgery for lung resection. Another factor affecting the diffusion of the robotic approach in thoracic surgery is the diffusion of uniportal thoracoscopy that has led to a critical review of the concept of mini-invasiveness, describing greater body preservation in patients undergoing lung surgery. Oncological results obtained with uniportal thoracoscopy also appear to be similar to those reported in open surgery (6). Despite these aspects, robotic supporters prefer it because of several advantages over VATS, including intuitive movements, tremor filtration, more degrees of manipulative freedom, motion scaling, and high definition stereoscopic vision. These advantages promise to make robotic surgery more accessible than VATS.

\section{Different robotic approaches to the lung}

The last generation robotic system was introduced in 2014. The advantages of this new system are a simpler docking, a more user-friendly design, a "port placement" menu and laser guidance. In addition, the thoracoscope has a digital end-mounted camera with autofocus for improved vision that does not require draping and can be placed onto any of the robotic arms. Lastly, the improved design of the arms allows placement of the ports relatively close together while still avoiding collision.

Different techniques of robotic approach to lung have been described in recent years. We here describe the different approaches used in robotic lung surgery: the two main approaches can be summarized in the anterior one with a utility incision (RAL) and in the complete port robotic lobectomy (CPRL) that does not include the use 
of a utility incision but uses $\mathrm{CO}_{2}$ during operation; other hybrid techniques have been described.

In 2005 Melfi and colleagues reported the first series of robotic lobectomy (7). In 2006 Park described a similar technique, where the port positions was similar to that used by the anterior VATS approach with a utility incision of $3-4 \mathrm{~cm}$ in the IV intercostal space on the mid axillary line, and uses two more trocars for the camera port and for the second instrument (4). This approach was modified by Veronesi et al. and described in the paper comparing open, muscle-sparing thoracotomy and robotic lobectomy (fourarm technique with a $3 \mathrm{~cm}$ utility incision) (8).

In 2011, Cerfolio described a new "closed" technique (9). This was a four-arm technique in which the four arms where positioned along the same intercostal space (usually the 7 th), between the mid-axillary and paravertebral lines, at the minimum distance of $9 \mathrm{~cm}$, with no utility incision.

In the same year Dylewski et al. (10) reported on 200 robotic resections using a three-arm completely port approach with $\mathrm{CO}_{2}$-induced pneumothorax (complete portal robotic lobectomy, CPRL).

Gharagozloo et al. (11) reported on another hybrid technique, composed by traditional thoracoscopy and robotics. Robotic arms were used for isolation of hilar elements and mediastinal lymph-node dissection, followed by stapling of the hilar structures using a manual VATS approach.

In the recent times the biggest innovation that has been made in the field of robotic surgery is represented by robotic endowrist staplers introduced in 2014. Stapler division of the hilar structures is considered one of the most important and potentially hazardous steps during a lobectomy. For some surgeons, the delegation of this task to the assistant is considered a risk. The use of the robotic stapler allows the surgeon to operate in absolute autonomy managing by himself the vascular section and seems to be safe and effective. The operating surgeon's ability to control the stapler from the console represents a critical technical advancement, as it can allow surgeons with limited assistance to explore robotic lung resection and perhaps transition from open or video-assisted lobectomy (12).

\section{State of the art of robotic lung lobectomy techniques}

Since the beginning we have adopted an anterior approach with utility incision. This approach mainly differs from the CPRL for the type of approach to the hylum (anterior or posterior) and for the use of $\mathrm{CO}_{2}$ during the procedure.

In our opinion the presence of a utility incision is related to some advantages as the possibility of palpating the lung and removing the specimen from the same incision; in case of vascular bleeding it allows a rapid conversion, with the possibility of enlarging the same incision; it also offers a comfortable access of a sponge in the case of small bleeding and avoids one trocar incision compared to the complete portal technique $\mathrm{CO}_{2}$ is not routinely used in this techniques, but it is indicated in selected cases such as obesity, relaxation of the diaphragm, incomplete lung exclusion due to air trapping in COPD patients or problems with the tracheal tube (13).

The benefit of complete portal robotic procedures is the presence of $\mathrm{CO}_{2}$. This can be related to the potential advantage to avoid the cold $22{ }^{\circ} \mathrm{C}$ ambient air of the operating room interfering with the $37{ }^{\circ} \mathrm{C}$ temperature within the chest, preventing potential tissue desiccation and further inflammation; it is also useful because it helps to detach the pulmonary parenchyma showing better the hilar structures and increase the working place in the chest cavity.

The use of robotics in thoracic surgery has increased considerably over the last few years. Although no new technical variations have been described, the presence of more recent articles has resulted in confusion in terms used to describe different techniques. To solve this problem the American Association of Thoracic Surgeons Guideline Committee appointed an expert consensus writing committee to construct definitions and nomenclature for robotic thoracic surgery to describe the current and possible subsequent types of robotic operations performed in general thoracic surgery (14).

The results of this consensus statement give some definitions that may help the future article to be better classified. One of the issues attempts to define the differences between a complete portal approach from the utility incision: the consensus define a robotic portal (RP) operation as any operation that use ports only (incisions that are only as large as the size of the trocars placed in them), the air in the pleural space or chest cavity does not directly communicate with the ambient air in the operating room. Robotic operations that include a utility incision have been defined as robotic-assisted (RA) procedures.

At this regard the author proposes the following nomenclature: the first letter " $\mathrm{R}$ " should be used to identify a robotic procedure, the second letter should describe a portal $(\mathrm{P})$ or assisted $(\mathrm{A})$ procedure. The third letter(s)—what operation is being performed: " $\mathrm{L}$ " for 
lobectomy, "S" for segmentectomy, "W" for wedge, "P" for pneumonectomy and "SL" for sleeve lobectomy. The fourth letter should describe the number of robotic arms used. Thus, a completely portal lobectomy that uses 4 arms is a RPL-4; a robotic segmentectomy that is robot assisted and uses 4 arms would be RAS- 4 and. A robotic sleeve lobectomy that uses 4 arms with a utility incision is abbreviated RASL-4.

Although this new classification system goes to the direction of harmonized the classifications of different robotic procedures, some criticisms have been moved in the editorial of Abbas E. Abbas. He asked to identify one system that can resume all the robotic procedures made on the lung, pleura, chest wall, mediastinum, esophagus, and stomach (15). He also added that surgeons must be persuaded to change what they call their own operations and other thoracic and minimally invasive societies will have to support this nomenclature.

\section{Future direction for robotic thoracic surgery}

Beside indisputable technical advantages of robotic approach, there are still some doubts today about the clinical benefits of robotic approach in lung surgery compared to manual video-assisted surgery considering that both are minimally invasive approach. In addition to assess oncological benefits we should wait for longer follow up data. Preliminary results based on retrospective studies or meta-analysis seems to give an advantage in terms of fewer conversions compared to VATS and a greater number of removed lymph nodes and upstage (16). A recent metaanalysis shows a small but significant benefit in terms of postoperative mortality (17). Despite these initial positive results, the high costs associated with the procedure deserve a higher level of evidence, hopefully based on randomized trials, to justify diffuse adoption.

In order to fill the gap, we started a multicentric randomized study to compare the results of robotic versus manual video-thoracoscopic lung resection in patients with non-small cell lung cancer in stage I and II, in terms of perioperative outcome, oncological radicality and quality of life (Trial Gov NCT02804893).

Regarding the costs we recently analyzed retrospectively 103 consecutive patients who underwent a lobectomy or a segmentectomy for clinical stage I or II NSCLC with three different approaches: thoracotomy, robotic and VATS. We analyzed clinical, surgical data and costs. Our results showed that although the costs of robotic approach was higher compared to other techniques, in one system of public health reimbursement, our hospital was able to make a profit (submitted data). Preliminary data on clinical outcome showed also that robotic surgery for early lung cancer was associated with shorter stay and more extensive lymph node dissection than VATS and open surgery. Duration of surgery was shorter for robotic than VATS.

Since its introduction into the market robotic system was produced by a single company that had maintained the costs high. The global crisis over the last decade associated with these high costs has not facilitated the spread of this highly demanding technology from an economic point of view by the organization of healthcare facilities. The high technology associated with the robot probably will never equal the cost to traditional thoracoscopy. However, in the coming years new producers will launch on the market new surgical robots (probably already in 2018). The improvement in the global economic situation associated with the entry of competitors that will certainly lead to lower costs can facilitate the diffusion of the technology (3).

The first desirable goals for robotic surgery are the entry of the "single site" technology in thoracic surgery. So far, the available technological platforms and the results of operations in urological and obstetrical pathology are not yet sufficient and exhaustive (18), but an imminent future is likely to occur in response to more and more frequent use of uniportal manual thoracoscopy.

The second goal could be the incorporation of a technology capable of receiving tactile feedback whose absence in robotic surgery has generated much skepticism.

The last important step in robotic surgery should be in our opinion the integration of the preoperative imaging information with intraoperative anatomical information obtained in real time by robotic visual system. The augmented reality (AR) can allow the surgeon to locate blood vessels or other structures that are not directly visible, and which previously could only be appreciated by palpation. It will also require a radical change in operating room practice and mindset.

\section{Acknowledgements}

The Umberto Veronesi Foundation is gratefully acknowledged for fellowship to Pierluigi Novellis.

\section{Footnote}

Conflicts of Interest: G Veronesi is a consultant for ABI 
Medica SpA and Medtronic. The other authors have no conflicts of interest to declare.

\section{References}

1. Jin R, Yang S, Wei G, et al. Robotic Thoracic surgery: left inferior lobectomy. AME Med J 2017;2:13.

2. Melfi FM, Menconi GF, Mariani AM, et al. Early experience with robotic technology for thoracoscopic surgery. Eur J Cardiothorac Surg 2002;21:864-8.

3. Novellis P, Alloisio M, Vanni E, et al. Robotic lung cancer surgery: review of experience and costs. J Vis Surg 2017;3:39.

4. Park BJ, Flores RM, Rusch VW. Robotic assistance for video-assisted thoracic surgical lobectomy: technique and initial results. J Thorac Cardiovasc Surg 2006;131:54-9.

5. Swanson SJ, Miller DL, McKenna RJ Jr, et al. Comparing robot-assisted thoracic surgical lobectomy with conventional video-assisted thoracic surgical lobectomy and wedge resection: results from a multihospital database (Premier). J Thorac Cardiovasc Surg 2014;147:929-37.

6. Ismail M, Swierzy M, Nachira D, et al. Uniportal videoassisted thoracic surgery for major lung resections: pitfalls, tips and tricks. J Thorac Dis 2017;9:885-97.

7. Melfi FM, Ambrogi MC, Lucchi M, Mussi A. Video robotic lobectomy. Multimed Man Cardiothorac Surg 2005;2005:mmcts.2004.000448.

8. Veronesi G, Galetta D, Maisonneuve P, et al. Four-arm robotic lobectomy for the treatment of early-stage lung cancer. J Thorac Cardiovasc Surg 2010;140:19-25.

9. Cerfolio RJ, Bryant AS, Minnich DJ. Starting a robotic

Cite this article as: Novellis $\mathrm{P}$, Alloisio M, Cariboni U, Veronesi G. Different techniques in robotic lung resection. J Thorac Dis 2017;9(11):4315-4318. doi: 10.21037/ jtd.2017.10.69 program in general thoracic surgery: why, how, and lessons learned. Ann Thorac Surg 2011;91:1729-36; discussion 1736-7.

10. Dylewski MR, Ohaeto AC, Pereira JF. Pulmonary resection using a total endoscopic robotic video-assisted approach. Semin Thorac Cardiovasc Surg 2011;23:36-42.

11. Gharagozloo F, Margolis M, Tempesta B, et al. Robotassisted lobectomy for early-stage lung cancer: report of 100 consecutive cases. Ann Thorac Surg 2009;88:380-4.

12. Pearlstein DP. Robotic Lobectomy Utilizing the Robotic Stapler. Ann Thorac Surg 2016;102:e591-3.

13. Veronesi G, Novellis P, Voulaz E, et al. Robot-assisted surgery for lung cancer: State of the art and perspectives. Lung Cancer 2016;101:28-34.

14. Cerfolio R, Louie BE, Farivar AS, et al. Consensus statement on definitions and nomenclature for robotic thoracic surgery. J Thorac Cardiovasc Surg 2017; 154:1065-1069.

15. Abbas AE. New nomenclature for robotic-assisted thoracic surgery also gets rid of RATS. J Thorac Cardiovasc Surg 2017;154:1070-1.

16. Mungo B, Hooker CM, Ho JS, et al. Robotic Versus Thoracoscopic Resection for Lung Cancer: Early Results of a New Robotic Program. J Laparoendosc Adv Surg Tech A 2016;26:243-8.

17. Emmert A, Straube C, Buentzel J, et al. Robotic versus thoracoscopic lung resection: A systematic review and meta-analysis. Medicine (Baltimore) 2017;96:e7633.

18. Nelson RJ, Chavali JSS, Yerram N, et al. Current status of robotic single-port surgery. Urol Ann 2017;9:217-22. 